University of Nebraska - Lincoln

DigitalCommons@University of Nebraska - Lincoln

$11-2013$

\title{
Quasi-monoenergetic and tunable X-rays from a laser-driven Compton light source
}

\author{
Nathan D. Powers \\ University of Nebraska-Lincoln, ndp5@byu.edu \\ Isaac A. Ghebregziabher \\ University of Nebraska - Lincoln, ighebregziabiher2@unl.edu \\ Grigory V. Golovin \\ University of Nebraska-Lincoln, ggolovin2@unl.edu \\ Cheng Liu \\ University of Nebraska-Lincoln, cliu8@unl.edu \\ Shouyuan Chen \\ University of Nebraska-Lincoln, schen6@unl.edu
}

See next page for additional authors

Follow this and additional works at: https://digitalcommons.unl.edu/physicsumstadter

Powers, Nathan D.; Ghebregziabher, Isaac A.; Golovin, Grigory V.; Liu, Cheng; Chen, Shouyuan; Banerjee, Sudeep; Zhang, Jun; and Umstadter, Donald P., "Quasi-monoenergetic and tunable X-rays from a laserdriven Compton light source" (2013). Donald Umstadter Publications. 92.

https://digitalcommons.unl.edu/physicsumstadter/92

This Article is brought to you for free and open access by the Research Papers in Physics and Astronomy at DigitalCommons@University of Nebraska - Lincoln. It has been accepted for inclusion in Donald Umstadter Publications by an authorized administrator of DigitalCommons@University of Nebraska - Lincoln. 


\section{Authors}

Nathan D. Powers, Isaac A. Ghebregziabher, Grigory V. Golovin, Cheng Liu, Shouyuan Chen, Sudeep

Banerjee, Jun Zhang, and Donald P. Umstadter 


\title{
Quasi-monoenergetic and tunable X-rays from a laser-driven Compton light source
}

\author{
N. D. Powers, I. Ghebregziabher, G. Golovin, C. Liu, S. Chen, \\ S. Banerjee, J. Zhang, and D. P. Umstadter \\ Department of Physics and Astronomy, University of Nebraska-Lincoln, Lincoln, Nebraska 68588, USA \\ Corresponding author - D. P. Umstadter, email donald.umstadter@unl.edu
}

\begin{abstract}
The maximum achievable photon energy of compact, conventional, Compton-scattering X-ray sources is currently limited by the maximum permissible field gradient of conventional electron accelerators. ${ }^{1,2}$ An alternative compact Compton X-ray source architecture with no such limitation is based instead on a high-field-gradient laser-wakefield accelerator. ${ }^{3-6}$ In this case, a single high-power (100 TW) laser system generates intense laser pulses, which are used for both electron acceleration and scattering. Although such all-laserbased sources have been demonstrated to be bright and energetic in proof-of-principle experiments, ${ }^{7-10}$ to date they have lacked several important distinguishing characteristics of conventional Compton sources. We now report the experimental demonstration of all-laser-driven Compton X-rays that are both quasi-monoenergetic ( $50 \%$ full-width at half-maximum) and tunable $(\sim 70 \mathrm{keV}$ to $>1 \mathrm{MeV})$. These performance improvements are highly beneficial for several important X-ray radiological applications. ${ }^{2,11-15}$
\end{abstract}

Synchrotron X-ray sources are used for scientific research worldwide because of their high brightness, tunability and narrow photon-energy bandwidth. However, synchrotrons are typically large devices (stadium-sized) because of the size of their component electron accelerator and undulator. More compact (room-sized) synchrotron X-ray sources have recently been reported. ${ }^{1,2}$ These make use of an electromagnetic undulator operating on the principle of inverse-Compton scattering instead of a fixed-magnet undulator. The much higher frequency of optical laser light used in Compton scattering than in fixed-magnet arrays reduces the electron energy required to produce X-rays, which in turn reduces the required size of the electron accelerator. Even greater size reductions can be achieved when laser light is used to drive both the undulator and the electron accelerator. ${ }^{7-10,16}$

The radiofrequency (RF)-cavity-driven accelerator at Lawrence Livermore National Laboratory (LLNL) produces 478 $\mathrm{keV}$ X-ray beams with $12 \%$ spectral bandwidth, 16 ps inferred temporal duration, and a flux of $1 \times 10^{5}$ photons per shot, corresponding to $1.5 \times 10^{15}$ photons s $\mathrm{mm}^{-2} \mathrm{mrad}^{-2}$ (per $0.1 \%$ bandwidth) peak brilliance. Utilizing a laser-wakefield accelerator (LWFA) ${ }^{17,18}$ with an accelerating field gradient more than 1,000 times higher than conventional RF-cavity-driven accelerators (and thus much more compact), we now demonstrate the production of quasimonoenergetic X-rays that are 1,000 times brighter with 10 times more photons/shot. The increased brightness is due to the femtosecond pulse duration and micrometer-scale transverse size of LWFA electron beams and the X-rays they produce. This all-optical concept has been discussed theoretically ${ }^{3-6,19}$ and has been demonstrated in proofof-principle experiments. ${ }^{7-10}$ However, the X-rays produced thus far have had large bandwidths and fixed energy, even though narrow-bandwidth and tunable LWFA-generated electron beams have already been demonstrated. ${ }^{20-25}$ We now report X-rays from an LWFA-Compton light source that are not only bright and energetic, but also possess several other distinguishing characteristics of synchrotron light: narrow-bandwidth ( $\sim 50 \%$ full-width at half-maximum, FWHM) and central energy tunability over a wide range $(\sim 70 \mathrm{keV}$ to $.1 \mathrm{MeV})$.

In the experiment we made use of the $100 \mathrm{TW}, 800 \mathrm{~nm}$ Diocles laser at the University of Nebraska, Lincoln. ${ }^{26}$ The laser beam was divided into two synchronized light pulses by an optical beamsplitter. As shown schematically in Figure 1a, one pulse drives the LWFA, and the other acts as an undulator by scattering from the electrons (see Methods). After interacting with the laser pulse, the electron beam is swept out of the X-ray path by a dipole magnet and onto a charge-calibrated detector, which records the electron-beam energy spectrum and angular divergence for each shot (Figure 1b). The transverse spatial profile of the forward-directed X-ray beam is recorded by a calibrated detector (Figure 1c) placed outside the vacuum system.

The X-ray spectral bandwidth was directly measured by comparing the X-ray transmittances through a set of four Ross-filter ${ }^{27}$ pairs, with each pair composed of two elements of similar atomic number. The thickness of each pair (Table 1) was designed such that the transmission curves closely match for all photon energies outside of the two K-edges. The difference in transmitted signal through each pair (Supplementary Figure 1) was used to determine the number of photons with energies between the K-edges (see Methods). The filters were arranged in a checkerboard pattern to allow accurate reconstruction of the unfiltered X-ray spatial profile and to allow for multiple measurements across the beam's profile. Figure 2 shows the X-ray energy distribution, measured using filters contained within $4.5 \mathrm{mrad}$ of the beam center (slightly smaller than the 10 mrad FWHM beam divergence). The X-ray spectrum was centred at $66 \pm 7 \mathrm{keV}$, with an energy spread of $\sim 50 \%$ FWHM (fitted with a Gaussian profile). By virtue of being peaked, rather than exponentially decaying with increasing energy (as in previous all-laser-driven Compton experiments ${ }^{7-10}$ ), this spectrum has the advantage of having far fewer low-energy photons.

Assuming a collimated electron beam and narrow-bandwidth counterpropagating laser, the fractional bandwidth of the on-axis Compton-scattered spectrum is $\Delta E_{\gamma} / E_{\gamma} \approx 2 \Delta E_{e} / E_{e}$ where $E_{e}$ and $\Delta E_{e}$ are the electron-beam average energy and energy spread, and $E_{\gamma}$ and $\Delta E_{\gamma}$ are the $\mathrm{X}$-ray beam average energy and energy spread, respectively. The measured electronbeam spectrum peaks at $55 \pm 3 \mathrm{MeV}$ (Figure 2, inset), with a $22 \%$ (FWHM) energy spread (the spectral shape was well represented by a Gaussian fit) and $23 \mathrm{pC}$ beam charge. The $\sim 6 \%$ increase in the measured X-ray energy spread ( $\sim 50 \%)$, compared with the analytical prediction $(\sim 44 \%)$, is attributable to 


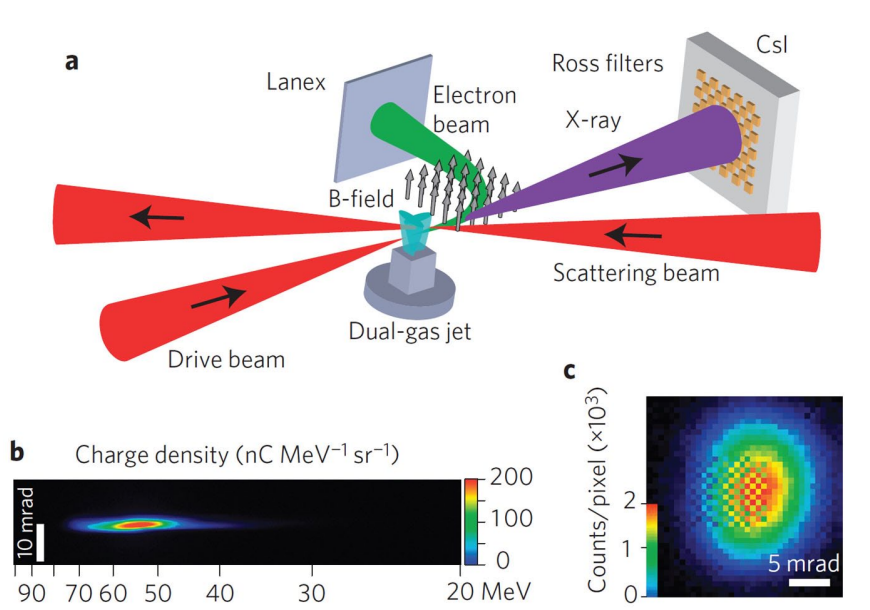

Figure 1. X-ray generation and detection. a) Experimental set-up (not to scale). Electrons (green) are accelerated by the interaction of a drive laser beam (red, from left to right) with a plasma created within the plume of a dual-stage gas jet and then deflected to a LANEX screen. A second laser pulse (red, from right to left) scatters from the electron beam after the accelerator. The X-ray beam (purple) is recorded by a CsI detector after passing through Ross filters arranged in a chequerboard pattern. b) Raw LANEX image of the electron-beam spectrum. c) Processed X-ray profile imaged through Ross filters.

Table 1. Ross-filter pairs used for X-ray spectral measurement.

\begin{tabular}{crr}
\hline Ross pair & Filter 1 & Filter 2 \\
\hline 1 & Dy $(96 \pm 1 \mathrm{~mm})$ & Sn $(234 \pm 2 \mathrm{~mm})$ \\
& Cu $(50 \pm 1 \mathrm{~mm})$ & Cu $(25 \pm 1 \mathrm{~mm})$ \\
2 & Ta $(41 \pm 1 \mathrm{~mm})$ & Dy $(96 \pm 1 \mathrm{~mm})$ \\
& $\mathrm{Cu}(16 \pm 1 \mathrm{~mm})$ & Cu $(50 \pm 1 \mathrm{~mm})$ \\
3 & Au $(30 \pm 1 \mathrm{~mm})$ & Ta $(41 \pm 1 \mathrm{~mm})$ \\
& Cu $(6 \pm 1 \mathrm{~mm})$ & Cu $(16 \pm 1 \mathrm{~mm})$ \\
4 & $\mathrm{~Pb}(48 \pm 1 \mathrm{~mm})$ & Au $(30 \pm 1 \mathrm{~mm})$ \\
& & Cu $(6 \pm 1 \mathrm{~mm})$ \\
\hline
\end{tabular}

Errors represent the sum of both measurement errors, determined from device sensitivity and variation in filter thickness across the sample.

contributions from the laser spectral bandwidth, scattering angle, divergence and laser ponderomotive force, all of which in our case were small in comparison to the energy width contributed by the electron beam.

The experimental measurement of the X-ray bandwidth was compared with the predictions of a previously benchmarked numerical model ${ }^{6}$ (Supplementary Methods). The model simulates the scattered X-ray spectrum from the measured electron-beam characteristics (that is, the energy spectrum and energy-dependent divergence of Figure 1b), represented by a six-dimensional phase-space distribution, and the laser parameters (150 fs laser scattering pulse duration; focused intensity of $2 \times 10^{17} \mathrm{~W} \mathrm{~cm}^{-2}$ ). The model predicts an on-axis $\mathrm{X}$-ray spectrum peaked at $71 \mathrm{keV}$. Off-axis, the X-ray central energy decreases smoothly with increasing polar angle. Averaging the spectrum over the polar angles measured in the experiment results in an additional redshift, with no significant change in the spectral width. The simulated spectrum, including contributions from all photons within the area used for Ross-filter analysis, predicts an averaged spectrum (Figure 2) that peaks at $64 \mathrm{keV}$, with $50 \%$ fractional bandwidth. Thus, the energy, the bandwidth and the number of photons within the measured region $\left(4.6 \times 10^{5}\right.$ simulated compared to $3 \times 10^{5}$ measured) are all well within the uncertainty of the measurement.

The central X-ray energy was tuned over one order of magnitude by tuning the energy of the LWFA electron beams from

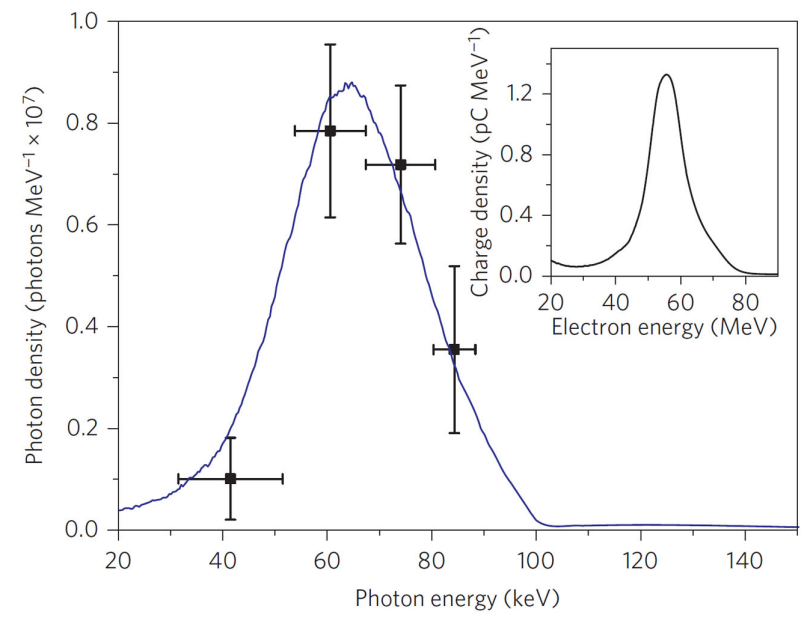

Figure 2. X-ray bandwidth measurement. X-ray spectral distribution (black squares) measured, for a single shot, by the Ross-filter pairs. Horizontal error bars represent the spectral width of the filter pair; vertical error bars represent the measurement error, including uncertainty in the filter thickness, crosstalk between voxels, and filter bandgap leakage. The simulated X-ray spectrum (solid line), obtained from the experimentally measured electron-beam spectrum (inset), is normalized by dividing by a factor of 1.4.

$\sim 50 \mathrm{MeV}$ to $300 \mathrm{MeV}$ (Figure 3). A different filter set and analysis method (see Methods) were required to measure the $X$ ray photon energy over this much larger range due to the limited working range of the Ross-filter technique (Supplementary Figure 2). Although the central energy could be measured by this method, the energy spread could not. For the $\sim 70$ shots in which the X-ray energy could be analyzed, the average $X$-ray central energy was observed to follow a quadratic scaling with the average electron-beam central energy, as the latter was tuned. This agrees with the theoretically predicted $E_{\gamma}=4 \gamma^{2} E_{\mathrm{L}}$ scaling for photons backscattered on-axis by a single electron, where $E_{\mathrm{L}}$ and $E_{\gamma}$ are the scattering laser and scattered $\mathrm{X}$-ray photon energies, respectively, and $\gamma$ is the relativistic Lorentz factor of the electron.

The reproducibility and stability of the X-rays were also computed, but using a larger data set. X-ray beams were observed on $>93 \%$ of shots (664 in total) when the scattering beam was present, illustrating the reproducibility of X-ray generation. For $56 \%$ of these shots, quasi-monoenergetic electron beams (energy spread $\lesssim 25 \%$ ) were measured. From the above experimental results and theoretical analysis, we can infer that the X-rays on these shots were also quasi-monoenergetic (energy spread $\lesssim 50 \%$ ). The measured electron energy spread, and thus inferred X-ray energy spread, was found to be most stable for the lowest and highest electron energy settings, where $>74 \%$ of the beams were quasi-monoenergetic (Supplementary Figure 3).

The X-ray photon number was stable to within $60 \%$ of its average value, $1.7 \times 10^{6}$ (Supplementary Figure 4). The photon number, and thus photon flux, depends on three factors: scattering-laser beam intensity, degree of beam overlap and electron-beam charge. The stability was dominated by the stability of the electron-beam charge, which for this data set was stable to within $45 \%$ of its average value, $29 \mathrm{pC}$. Greater control of the flux can be achieved by stabilizing the electron-beam charge and adjusting the scattering-beam intensity.

The all-laser-driven architecture has several advantages. Besides a small overall device size, the electron and scattering beam parameters are also well matched in size, and the pulses are well synchronized. These characteristics lead to high peak X-ray brightness: $\sim 3 \times 10^{18}$ photons $\mathrm{s}^{-1} \mathrm{~mm}^{-2} \mathrm{mrad}^{-2}$ (per $0.1 \%$ 

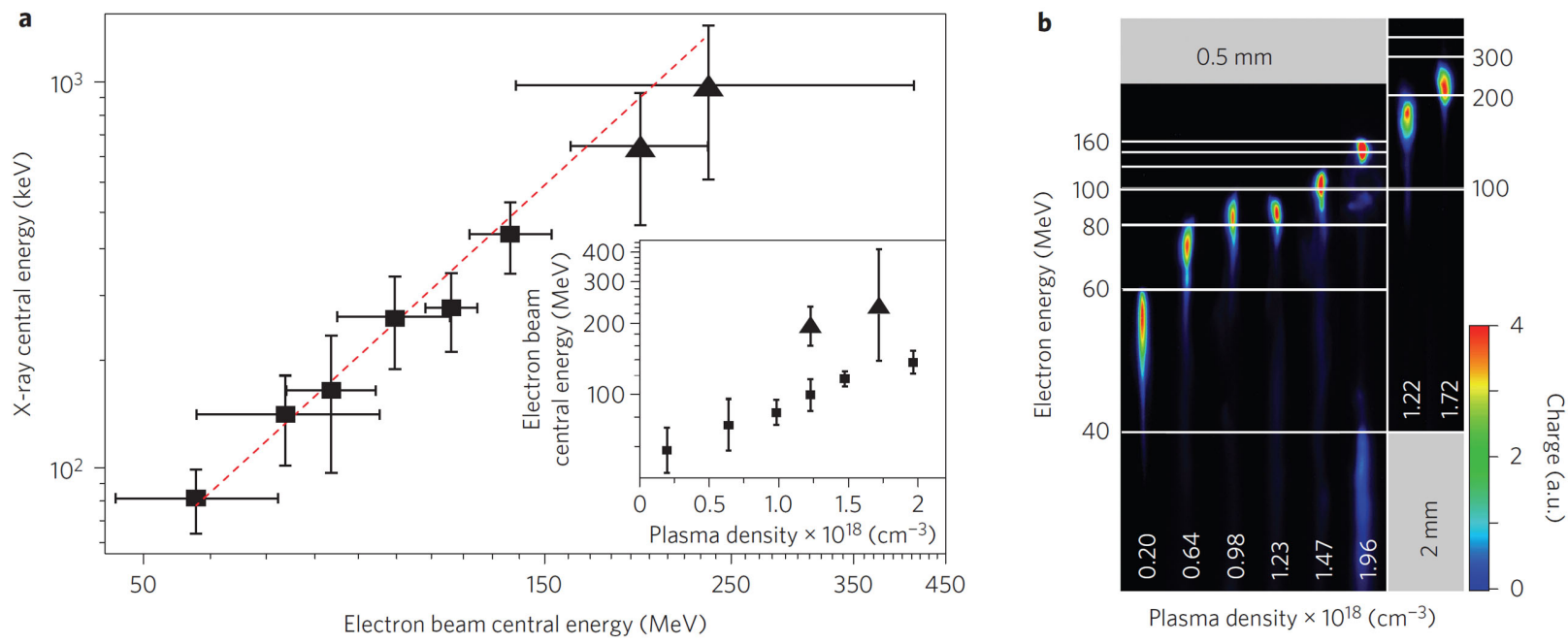

Figure 3. X-ray energy tuning. a) Measured X-ray central energy, plotted versus the measured electron-beam central energy, is consistent with a $4 \gamma^{2}$ scaling (red dashed line). The same data set, plotted versus plasma density (inset), illustrates that the electron-beam energy was controlled by adjusting the plasma density of two separate accelerator jets. For a given accelerator-jet length ( $0.5 \mathrm{~mm}$, black squares; $2 \mathrm{~mm}$, black triangles), the plasma density of the injectorjet was fixed $\left(1.7 \times 10^{18} \mathrm{~cm}^{-3}\right.$ and $2.9 \times 10^{18} \mathrm{~cm}^{-3}$, respectively). Each point is the average of, from left to right, $25,8,8$, $3,5,7,8$ and 5 selected shots (see Methods) taken with the same gas-jet parameters. Error bars represent $80 \%$ confidence intervals, calculated using the $t$-distribution of measurements of the set. $\mathbf{b}$ ) Individual electron-beam spectra from this data set were chosen to best represent the average electron-beam energy of the set. The beams are quasimonoenergetic across the entire tunable range.

bandwidth) for $70 \mathrm{keV}$ beams from Figure 2, and $\sim 1 \times 10^{19}$ for $1 \mathrm{MeV}$ beams from Figure 3. The brightness was calculated using the measured beam divergence and photon number, and assuming a $30 \mathrm{fs}$ X-ray pulse duration and $6 \mu \mathrm{m}$ (root mean square, r.m.s.) X-ray source size. ${ }^{10}$ The $1 \mathrm{MeV}$ beam brightness is comparable to previously reported polychromatic X-ray beams of similar energy. ${ }^{10}$

The improved X-ray characteristics reported here should expand the reach of compact all-laser-driven Compton X-ray sources. For instance, narrow X-ray bandwidth, relative to bremsstrahlung, significantly reduces the amount of unwanted radiation in medical imaging. Consequently, radiographic image quality is improved, ${ }^{12}$ patient risk is decreased ${ }^{11,13}$ and beam-hardening effects are reduced. ${ }^{2}$ Additionally, photon energy-tunability can enhance radiographic imaging. ${ }^{12}$

Further improvements in X-ray performance can be expected by incorporating techniques used in conventional Compton sources and/or advances in LWFA technology. For instance, by using either higher scattering photon energy (achieved by harmonic conversion) or higher LWFA electron energy, 25, 28 the X-ray energy will soon exceed the threshold for nuclear photo-disintegration $(\sim 10 \mathrm{MeV}) \cdot{ }^{14,15}$ Similarly, lower-bandwidth X-rays will result from using lower-bandwidth electron beams. ${ }^{20-23}$ Finally, the control and stability of the X-ray energy will be substantially improved by recent advances in the degree of control and stability achieved for LWFA electron energy. ${ }^{24}$

\section{Methods}

Laser. The drive beam was operated from 1.3 to $1.7 \mathrm{~J}$. Spectral- and spatial-phase correction optimized the beam characteristics. ${ }^{26}$ The resulting 33 fs pulse was focused with an $f / 14$ parabolic mirror to a $21 \mu \mathrm{m}$ (FWHM) Gaussian focal spot. The $150 \mathrm{fs}, 130-170 \mathrm{~mJ}$ scattering beam was focused with a thin positive lens ( $f=1,000 \mathrm{~mm}$ ) to $17 \mu \mathrm{m}$ (FWHM).

Undulator parameters. The electron beam and scattering laser were spatiotemporally overlapped $1.5 \mathrm{~mm}$ downstream of the accelerator exit at a $170^{\circ}$ angle (nearly backscattering). The laser had $N=56 \mathrm{cy}-$ cles, and a strength parameter of $a_{0}=0.3$.

Accelerator parameters. We used a dual-stage gas jet accelerator consisting of an injector stage of a 99:1 He: $\mathrm{N}_{2}$ mixed gas mixture ${ }^{29,30}$ and an accelerator stage of pure He. The central energy was increased, within a given range, by increasing the plasma density of the accelerator stage. Using a longer accelerator stage shifted the tunable energy range higher. The plasma density of the $0.5 \mathrm{~mm}$ acceleration stage was scanned from $2 \times 10^{17} \mathrm{~cm}^{-3}$ to $2 \times 10^{18} \mathrm{~cm}^{-3}$. For the two highest-energy data points shown in Figure 3, a $2 \mathrm{~mm}$ acceleration stage density was scanned from $1.2 \times 10^{18} \mathrm{~cm}^{-3}$ to $1.7 \times 10^{18} \mathrm{~cm}^{-3}$. A future publication will provide further details on the accelerator.

Detection. The electron spectrometer consisted of a $19 \mathrm{~cm}, 0.8 \mathrm{~T}$ magnet and a $\mathrm{Gd}_{2} \mathrm{O}_{2} \mathrm{~S}$ : Tb screen (LANEX) imaged by a 12-bit charge-coupled device (CCD) camera. A magnetic field map was used to calculate the electron deflection versus energy. The LANEX was placed outside the X-ray beam path to allow simultaneous measurement of the $X$-rays and electrons.

The X-ray detector was a $40 \times 40$ voxel array of 1 -mm-square, 1-cm-deep CsI crystals imaged by a 14-bit electron multiplying CCD camera. All voxels were separated by 0.2 -mm-wide epoxy. Crosstalk between voxels was measured using an X-ray tube source.

X-ray spectral measurement (Ross filters). Each filter was cut into a $1.2 \mathrm{~mm}$ square and aligned to a single detector voxel. The X-ray beam profile in filtered regions was reconstructed by a two-dimensional biharmonic spline interpolation. A transmittance map was produced by dividing the measured signal by the reconstructed one.

The signal difference between two filters of a Ross pair was used to calculate the number of photons within the measurement bandwidth of the pair $(\Delta E)$, written explicitly as

$$
\Delta S=\int_{0}^{\infty} X(E) \Omega(E) \Delta T(E) \mathrm{d} E
$$

where $E$ is photon energy, $\Omega(E)$ is the detector response, and $S$ and $T$ are the signal level and transmittance of individual filters, respectively. Assuming the X-ray photon density has a nearly linear slope within each Ross-pair bandwidth, the photon density can be written as

$$
X(E)=X^{\text {avg }}\left[1+\beta\left(E-E^{\text {avg }}\right) / \Delta E\right]
$$

where $E^{\text {avg }}$ is the average energy within the bandwidth and $\beta$ is the slope of the distribution within the bandwidth. The average photon density,

$$
X^{\text {avg }}=\Delta S \int_{E_{1}}^{E_{2}}\left[1+\beta\left(E-E^{\text {avg }}\right) / \Delta E\right] \Omega(E) \Delta T(E) \mathrm{d} E,
$$

is obtained by integrating across the Ross-pair bandwidth. We used $\beta$ $=0$ to calculate the X-ray photon density, but included uncertainty in the slope in the error analysis as

$$
\sigma \beta=\left|X_{\beta=\beta_{\max }}^{\text {avg }}-X_{\beta=-\beta_{\max }}^{\text {avg }}\right| / X_{\beta=0}^{\text {avg }}
$$

The upper limit of the slope $\left(\beta_{\max }=4\right)$ was estimated from simulations. Other factors contributing to the error bars include uncertainty 
in the filter thickness, crosstalk between voxels, and filter bandgap leakage. Measurements of a polychromatic and a higher energy beam are shown in Supplementary Figure 2, demonstrating the response of this technique to different spectral distributions.

Energy scaling analysis (continuum-attenuation filters). The central X-ray energy was measured with various broadband transmission filters (instead of $K$-edge filters from the Ross technique), including 1.6, 3 , and $12 \mathrm{~mm}$ aluminium, 0.5, 1, 1.5, 2, 3, 4.5, and $6 \mathrm{~mm}$ copper, 1.6 $\mathrm{mm}$ lead, and $0.175 \mathrm{~mm}$ uranium. The two highest-energy data points used $6.25 \mathrm{~mm}$ aluminium, $1.52 \mathrm{~mm}$ copper, and $6.24 \mathrm{~mm}$ lead. The filter edges were aligned across voxel rows and/or columns, with $>2$ voxels between neighboring filters, in crisscrossed-strip-filter, quadfilter, or step-filter arrangements.

Shot-to-shot fluctuation in beam centering on the filters limited the number of shots that could be analyzed. Because beam energy and pointing are not correlated, no bias was introduced by analyzing this subset of shots. The central energy of quasi-monoenergetic $X-$ ray beams $(<25 \%$ energy spread in the corresponding electron beam) was analyzed only if the X-rays were centered on the appropriate filter set, thus providing adequate signal-to-noise ratio for accurate profile reconstruction.

The beam profile in filtered regions was reconstructed by two-dimensional Gaussian fitting. The measured transmissions for each shot were compared to simulated transmissions for all combinations of $\mathrm{X}$ ray central energy and energy spread, and each combination represented a single test spectrum. The X-ray central energy and measurement uncertainty were determined from the set of test spectra with transmission values within the measurement error and a bandwidth $<150 \%$ of the analytical prediction. Some knowledge of the X-ray spectral shape, determined from the measured electron-beam energy distribution, was required to determine the central energy. Methodological consistency for all data points ensured accurate representation of the energy scaling. Only a lower limit of the central energy was determined for the two highest-energy sets.

Supplementary information is presented following the References.

Acknowledgments - The authors thank K. Brown, J. Mills, and C. Petersen for their contributions to the laser facility. The authors thank D. Haden and N. Cunningham from Nebraska Wesleyan University for their contributions to X-ray detector analysis. The authors thank C. Wilson, T. Anderson, and D. Alexander from the University of Nebraska-Lincoln Electrical Engineering department for precision-cutting of Ross filters. This material is based on work supported by the US Department of Energy (DE-FG02-05ER15663), the Defense Threat Reduction Agency (HDTRA1-11-C-0001), the Air Force Office for Scientific Research (FA 9550-08-1-0232 and FA9550-11-1-0157), the Department of Homeland Security (2007-DN-077-ER0007-02), the Defense Advanced Research Projects Agency (FA9550-09-1-0009), and USSTRATCOM (FA4600-12-D-9000). The views expressed here do not represent those of the sponsors. The authors declare no competing financial interests.

Author contributions - The experiments were conceived and designed by D.U., N.P., S.B., S.C., I.G., C.L. and G.G. Experiments were carried out by G.G., N.P., S.B., S.C., I.G., C.L. and J.Z. Data analysis was performed by G.G., I.G. and N.P. Materials and analysis tools were provided by G.G., I.G., S.B. and N.P. The manuscript was written by D.U. and N.P.

\section{References}

1. Albert, F. et al. Characterization and applications of a tunable, laser-based, MeVclass Compton-scattering gamma-ray source. Phys. Rev. 13, 070704 (2010).

2. Achterhold, K. et al. Monochromatic computed tomography with a compact laser-driven X-ray source. Sci. Rep. 3, 1313 (2013).

3. Catravas, P., Esarey, E. \& Leemans, W. P. Femtosecond X-rays from Thomson scattering using laser wakefield accelerators. Meas. Sci. Technol. 12, 1828-1834 (2001).

4. Umstadter, D., He, F. \& Lau, Y. Ultra-short wavelength X-ray system US patent 7,321,604 (2008).
5. Esarey, E., Schroeder, C. B. \& Leemans, W. P. Physics of laser-driven plasmabased electron accelerators. Rev. Mod. Phys. 81, 1229-1285 (2009).

6. Ghebregziabher, I., Shadwick, B. A. \& Umstadter, D. Spectral bandwidth reduction of Thomson scattered light by pulse chirping. Phys. Rev. 16, 030705 (2013).

7. Schwoerer, H., Liesfeld, B., Schlenvoigt, H. P., Amthor, K. U. \& Sauerbrey, R. Thomson-backscattered $\mathrm{X}$ rays from laser-accelerated electrons. Phys. Rev. Lett. 96, 014802 (2006).

8. Ta Phuoc, K. et al. All-optical Compton gamma-ray source. Nature Photon. 6, 308-311 (2012).

9. Mori, Y. et al. Head-on inverse Compton scattering X-rays with energy beyond $10 \mathrm{keV}$ from laser-accelerated quasi-monoenergetic electron bunches. Appl. Phys. Express 5, 056401 (2012).

10. Chen, $\mathrm{S}$. et al. MeV-energy $\mathrm{X}$ rays from inverse Compton scattering with laser-wakefield accelerated electrons. Phys. Rev. Lett. 110, 155003 (2013).

11. Boone, J. M. \& Seibert, J. A. A figure of merit comparison between bremsstrahlung and monoenergetic X-ray sources for angiography. J. X-ray Sci. Technol. 4, 334-345 (1994).

12. Carroll, F. E. Tunable monochromatic $X$ rays: a new paradigm in medicine. Am. J. Roentgenol. 179, 583-590 (2002).

13. Brenner, D. J. et al. Cancer risks attributable to low doses of ionizing radiation: assessing what we really know. Proc. Natl Acad. Sci. USA 100, 13761-13766 (2003).

14. Giulietti, A. et al. Intense g-ray source in the giant-dipole-resonance range driven by 10-TW laser pulses. Phys. Rev. Lett. 101, 105002 (2008).

15. Habs, D. et al. Vision of nuclear physics with photo-nuclear reactions by laser-driven gamma beams. Eur. Phys. J. D 55, 279-285 (2009).

16. Corde, $\mathrm{S}$. et al. Femtosecond $\mathrm{X}$ rays from laser-plasma accelerators. Rev. Mod. Phys. 85, 1-48 (2013).

17. Tajima, T. \& Dawson, J. M. Laser electron accelerator. Phys. Rev. Lett. 43, 267-270 (1979).

18. Esarey, E., Ride, S. K. \& Sprangle, P. Nonlinear Thomson scattering of intense laser pulses from beams and plasmas. Phys. Rev. E 48, 3003-3021 (1993).

19. Hartemann, F. V. et al. Compton scattering X-ray sources driven by laser wakefield acceleration. Phys. Rev. 10, 011301 (2007).

20. Geddes, C. G. R. et al. High-quality electron beams from a laser wakefield accelerator using plasma-channel guiding. Nature 431, 538-541 (2004).

21. Mangles, S. P. D. et al. Monoenergetic beams of relativistic electrons from intense laser-plasma interactions. Nature 431, 535-538 (2004).

22. Faure, J. et al. A laser-plasma accelerator producing monoenergetic electron beams. Nature 431, 541-544 (2004).

23. Brunetti, E. et al. Low emittance, high brilliance relativistic electron beams from a laser-plasma accelerator. Phys. Rev. Lett. 105, 215007 (2010).

24. Gonsalves, A. J. et al. Tunable laser plasma accelerator based on longitudinal density tailoring. Nature Phys. 7, 862-866 (2011).

25. Banerjee, S. et al. Generation of tunable, $100-800 \mathrm{MeV}$ quasimonoenergetic electron beams from a laser-wakefield accelerator in the blowout regime. Phys. Plasmas 19, 056703 (2012).

26. Liu, C. et al. Repetitive petawatt-class laser with near-diffractionlimited focal spot and transform-limited pulse duration. Proc. SPIE 8599, 859919 (2013).

27. Ross, P. A. A new method of spectroscopy for faint X-radiations. J. Opt. Soc. Am. 16, 433-436 (1928).

28. Leemans, W. P. GeV electron beams from a centimetre-scale accelerator. Nature Phys. 2, 696-699 (2006).

29. Liu, J. S. et al. All-optical cascaded laser wakefield accelerator using ionization-induced injection. Phys. Rev. Lett. 107, 035001 (2011).

30. Pollock, B. B. et al. Demonstration of a narrow energy spread, similar to $0.5 \mathrm{GeV}$ electron beam from a two-stage laser wakefield accelerator. Phys. Rev. Lett. 107, 045001 (2011). 


\section{Supplementary Information}

\section{Supplementary Methods}

Simulation code. The scattered radiation from a single electron was obtained by solving the relativistic equations of motion for an electron in the intense laser field. The total scattered radiation was then obtained by integrating over the $e$-beam phase space distribution and focal volume of the laser field. 


\section{Supplementary Figures}

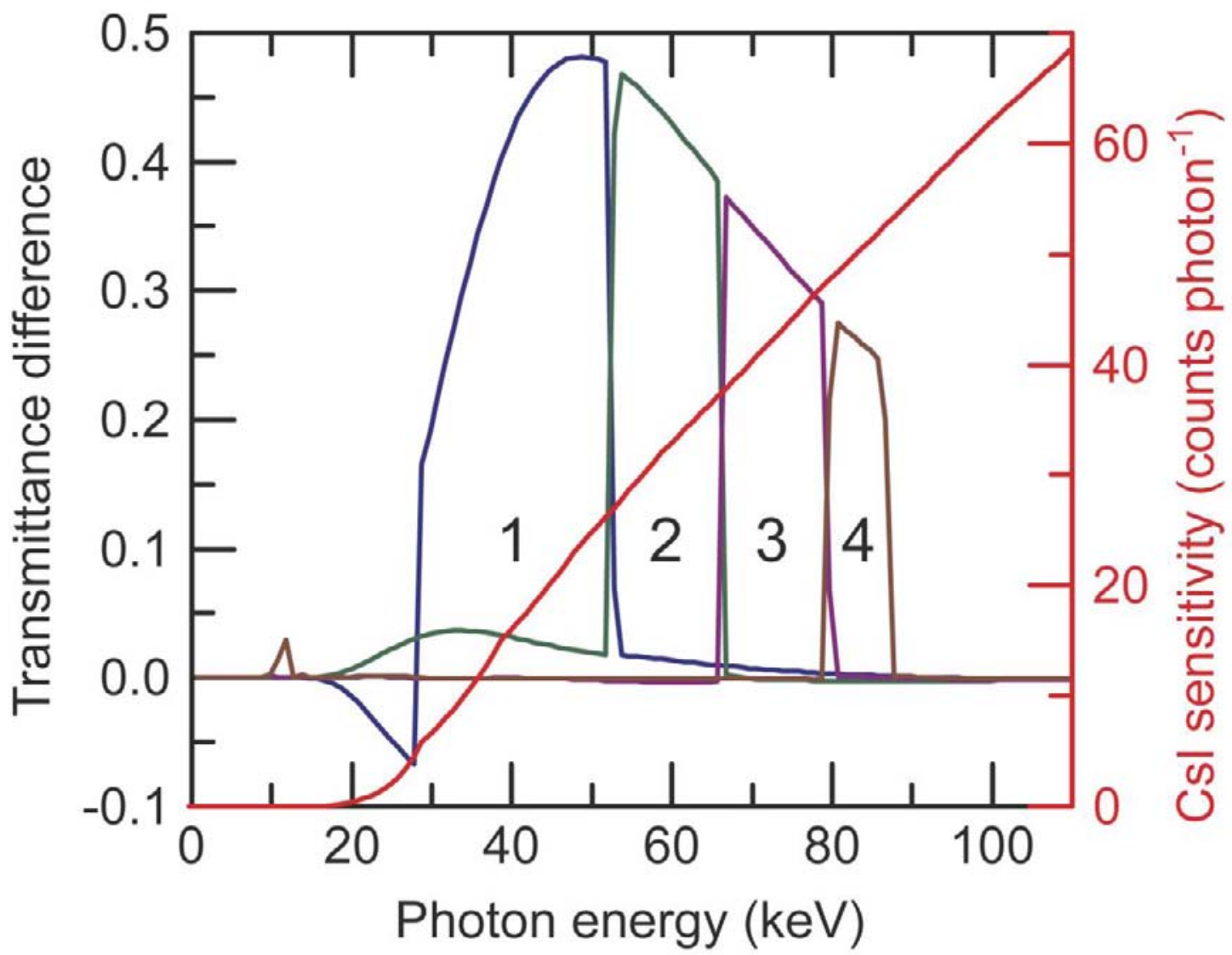

Figure S1: Ross-filter and CsI response curves. The transmission difference of filter pairs plotted (left axis) as a function of photon energy. Pair labeling matches that from Table 1. The CsI detector response curve (-) is also plotted (right axis) as a function of photon energy. 

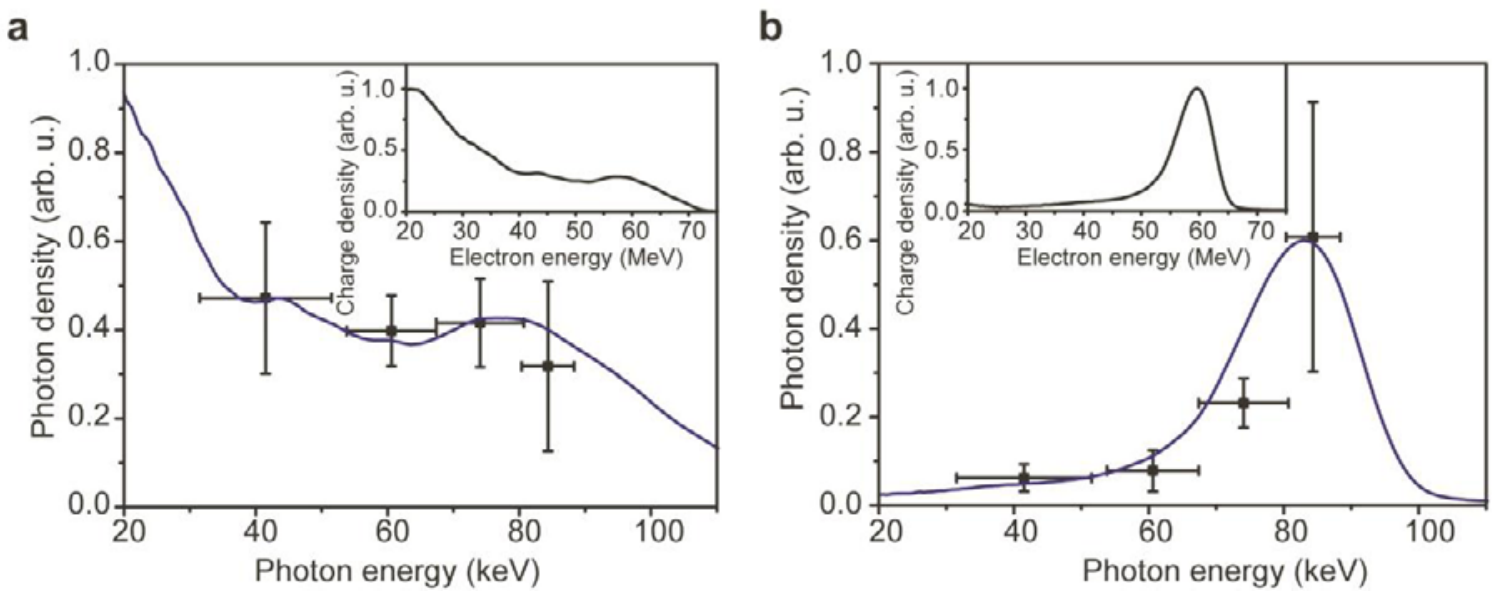

Figure S2: Comparison of x-ray spectra measured by the Ross-filter technique.

Spectral measurements obtained with Ross filters: (a) polyenergetic x-rays, and (b) highenergy monoenergetic x-rays. Experimental data points ( $\square$ ) have been normalized to the spectrum (-) produced by applying a $4 \gamma^{2} E_{L}$ scaling to the measured $e$-beam spectrum (inset). The results indicate that the technique can distinguish different spectral shapes. 


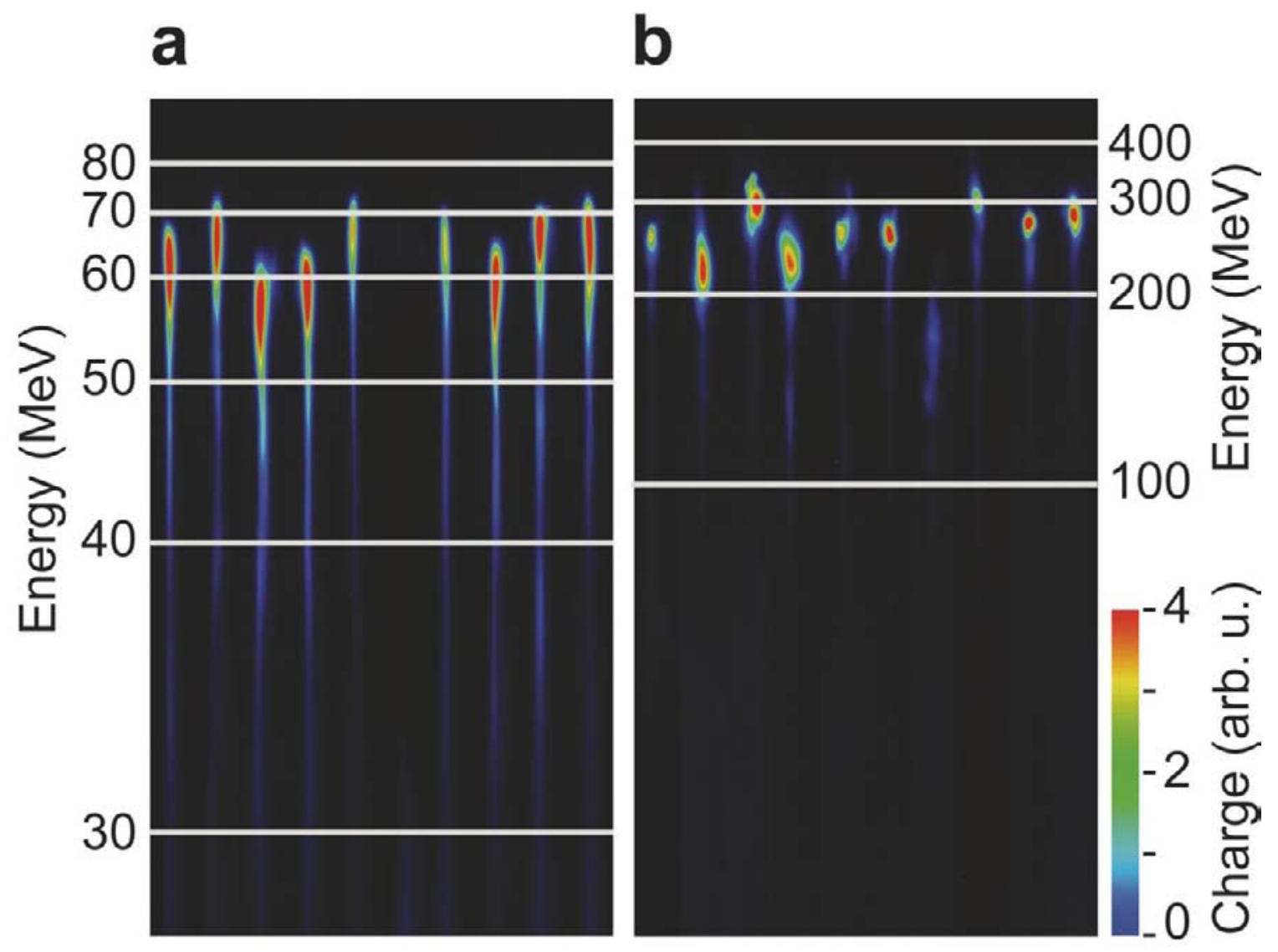

Figure S3: Control and stability of tunable quasi-monoenergetic $\boldsymbol{e}$-beams. LANEX

images of 10 consecutive shots taken with (a) plasma density: $2.0 \times 10^{17} \mathrm{~cm}^{-3}$; length: 0.5 $\mathrm{mm}$, and (b) plasma density: $1.7 \times 10^{18} \mathrm{~cm}^{-3}$; length: $2 \mathrm{~mm}$. These spectra are part of a larger set used, in the text, to calculate the x-ray beam reproducibility and stability for the lowest and highest energy tuning points of Fig. 3. Each series was chosen to best represent the statistics of the data set of which it is a part. The divergence of the beams is recorded in the horizontal axis while the energy is recorded on the vertical axis. 


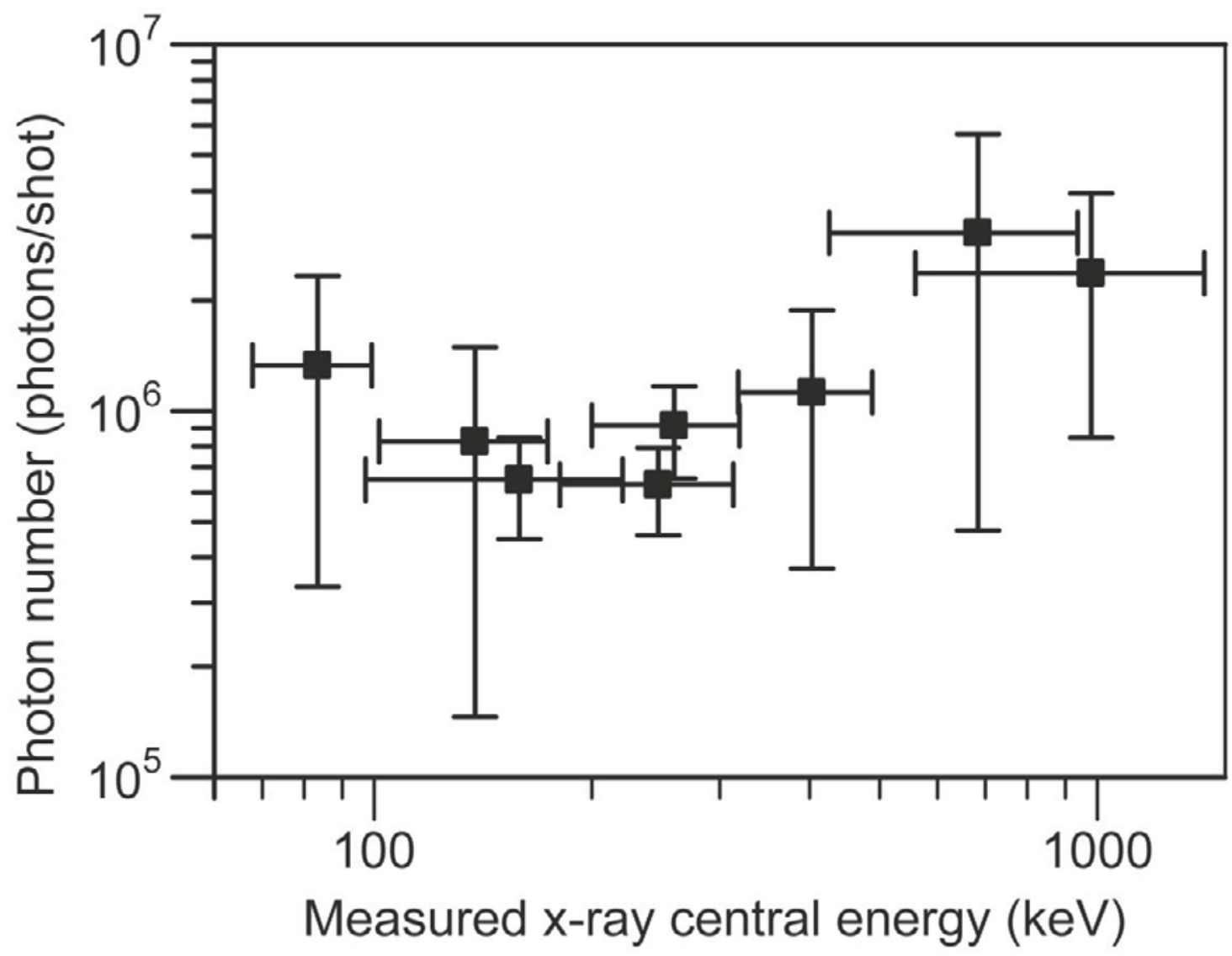

Figure S4: Photon number stability. The average photon number/shot, measured for each of the points shown in Fig. 3 of the main text, plotted as a function of the average central energy of the $\mathrm{x}$-ray beam. Vertical and horizontal error bars represent the $80 \%$ confidence intervals, calculated using $t$-distribution of shot-to-shot fluctuations in the measured values. Across an order-of-magnitude range of x-ray energy, the x-ray photon number is found to be stable to within $60 \%$ of its average value, $1.7 \times 10^{6}$. 